\title{
Editorial
}

\section{Et alia}

Dear Dr Green,

Thank you for offering us your case report on 'Acute renal failure resulting from liquorice ingestion'. It is a useful report and worthy of publication.

We are getting a bit sensitive about the number of authors who are alleged to have written short case reports. I know well the sort of pressures that cause one to include a mass of names on a paper; I also know that one has to resist them as tactfully as one can. We had decided that more than five names for a Short Report was inappropriate unless there were exceptional circumstances. Perhaps you could let me know if there are exceptional circumstances.

We are not just being awkward for the sake of it. A multiplicity of authors' names causes a multiplicity of work for subsequent indexers and persons who refer to the paper.

Yours sincerely,

In recent months several authors have received similar letters from us, and it may be helpful for other authors to consider the background.

In 1970 the mean number of authors for each original article published in the Archives was $2 \cdot 6$. By 1979 it had risen to 3.9. Ten years ago it was very rare for a paper to have more than 5 authors. Last year it was common for us to be offered papers with 8 or more co-authors and we did publish a short case report bearing the names of 13 authors (the cynic could calculate that each had written rather less than 2 sentences).

It would be good to imagine that the increased number of authors results from a blending of fertile scientific minds and an increasingly multidisciplinary approach. It rarely does. Too often it seems to be a case of mentioning everyone in the department, anyone who has said 'hello' to the child, or performed any investigation, however routine.
We know the important contributions made to the investigation and management of children by numerous medical and nonmedical workers, but believe that a scientific paper in our journal should bear the names of those who have done the special scientific and writing work for that paper, rather than anyone who has merely done their routine (and valuable) work. The acknowledgement section at the end allows the authors to thank other colleagues.

Each additional author causes extra work for the indexer and for anyone who uses the reference. A long list of names clutters up title pages and prevents all the names of the contributors appearing in their rightful place on the front cover. It is unfair to the main originators of the work, whose contribution appears diluted. We respectfully suggest that scientific stowaways and other hangers-on be jettisoned; we will continue to ask lead authors to justify the inclusion of more than 4 other co-authors. 